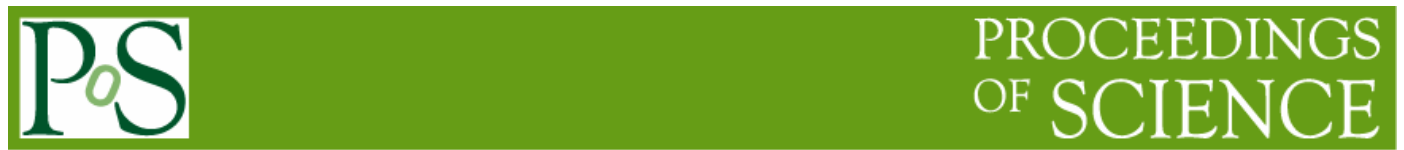

\title{
The Tully-Fisher relation of spiral galaxies
}

\section{Irina Yegorova*}

SISSA

Via Beirut 2/4, Italy

E-mail: yegorovaesissa.it

\section{Paolo Salucci}

SISSA

Via Beirut 2/4, Italy

E-mail: salucciesissa.it

The Tully-Fisher (TF) relation is an empirically establish correlation between the luminosity of spiral galaxy and its rotational velocity (Tully-Fisher, 1977). We used the Tully-Fisher relation in order to probe the dark matter (DM) distribution in the optical regions of spiral galaxies. We investigated a sample of rotation curves that includes 957 galaxies. The new technique applied takes advantages on the full knowledge of the galaxy rotation curves.

BDMH2004 - Baryons in Dark Matter Halos

5-9 October 2004

Novigrad, Croatia

\footnotetext{
${ }^{*}$ Speaker
} 
The Tully-Fisher (TF) relation has two important properties. First, it is gives possibility to obtain cosmological distances. Second, it can be used to study the dynamical properties and evolution of galaxies. We used the TF-relation to probe the dark matter (DM) distribution in the optical regions of spiral galaxies.

We investigated a sample of rotation curves (Persic, Salucci, 1995) that includes 957 galaxies (see also Mathewson, Ford 1996). The new technique we applied takes advantage of the full knowledge of the rotation curves of galaxies. More specifically we have established several new Tully-Fisher like relations, each one related to a different radius, namely at the following fixed fractions of the optical radius $\mathrm{R}_{\mathrm{opt}}$ (corresponding to about three length scales), i.e. at: $0.2,0.4,0.6,0.8,1.0,1.2$. The standard TF-relation is evaluated only at just one radius, usually about $\mathrm{R}_{\mathrm{opt}}$.
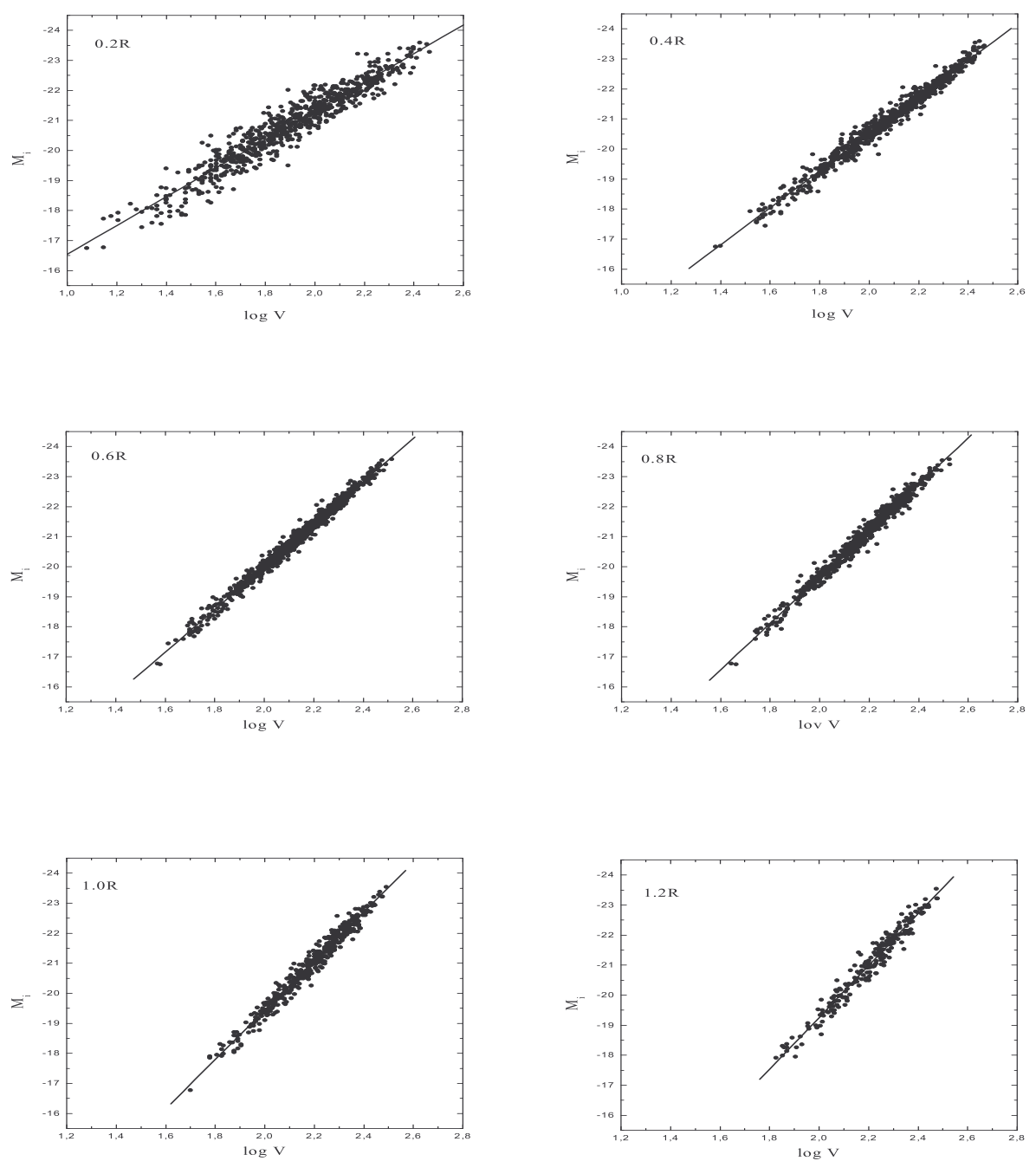

Figure 1. Sequence of the TF-relations at different radii. 
The thorough analysis of our data, which includes 15000 independent measurements, is done by means of the linear regression models $M b_{i}=a_{i}+b_{i} \log V\left(R_{i}\right)$, where the parameters $a_{i}$ and $b_{i}$ are found by least squares.

In the Fig. 1 we plot the TF-relations at the above specified radii and in Table 1 we present the main characteristics.

\begin{tabular}{|l|l|l|l|l|l|l|}
\hline $\mathrm{R} / \mathrm{R}_{\text {opt }}$ & $\begin{array}{l}\text { zero } \\
\text { point }\end{array}$ & error & slope & error & $\mathrm{SD}$ & $\mathrm{N}$ \\
\hline 0.2 & -11.78 & 0.103 & -4.77 & 0.054 & 0.366 & 739 \\
\hline 0.4 & -8.207 & 0.068 & -6.141 & 0.033 & 0.185 & 786 \\
\hline 0.6 & -5.769 & 0.063 & -7.102 & 0.029 & 0.146 & 794 \\
\hline 0.8 & -4.22 & 0.09 & -7.718 & 0.042 & 0.17 & 657 \\
\hline 1.0 & -8.2 & 0.146 & -8.197 & 0.067 & 0.208 & 447 \\
\hline 1.2 & -1.98 & 0.261 & -8.639 & 0.118 & 0.253 & 226 \\
\hline
\end{tabular}

column 1 - isophotal radius column 2 - intercept value (a) column 3 - standard error of a column 4 - slope value (b) column 5 - standard error of $b$ column 6 - scatter column 7 - number of points

Table 1. The TF-relations at different radii.

In the Fig. 2 we present the same result as in the Fig. 1 (TF-relations at different isophotal radii). Each colour corresponds to a different radius.

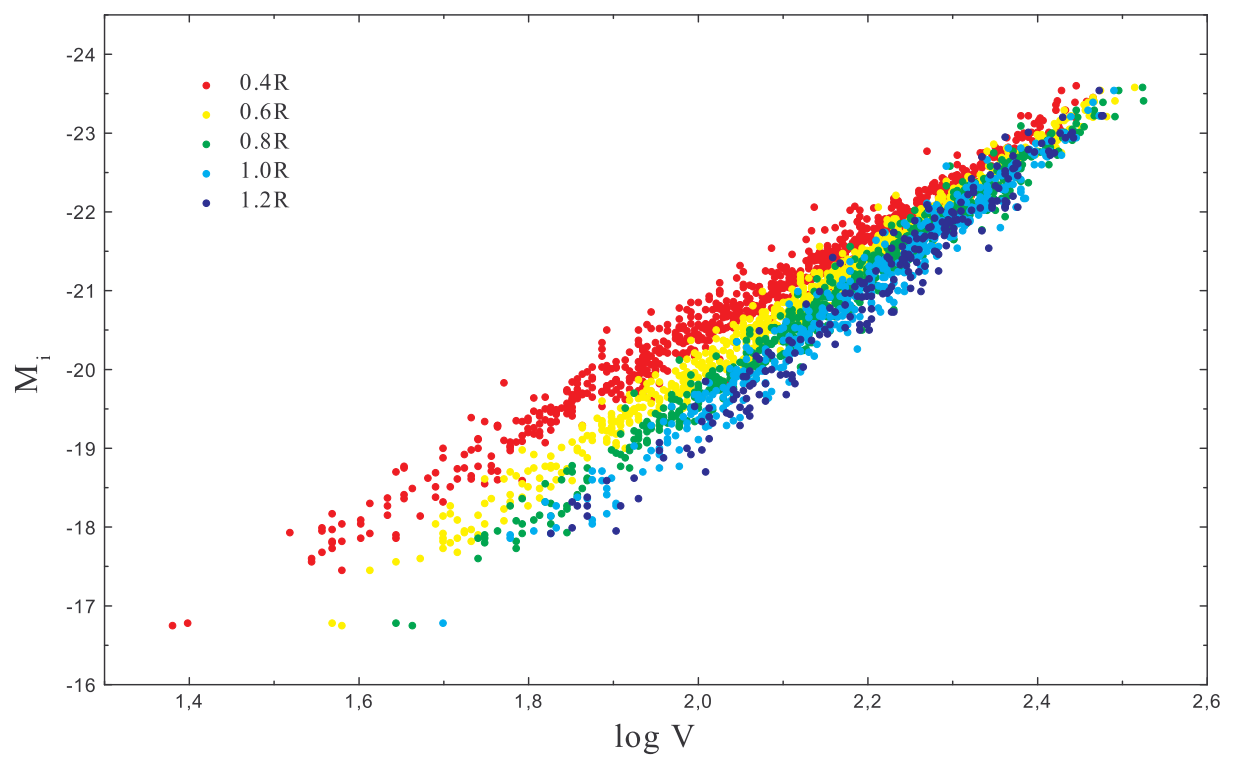

Figure 2. The TF-relations at different isophotal radii. 
In the Fig. 3 we show the scatter of these TF-relations as a function of their reference radius $R_{\mathrm{i}}$. Notice that at $0.2 \mathrm{R} / \mathrm{R}_{\mathrm{opt}}$ the scatter is very large, because of the close adjacency to the bulge, but otherwise, it is always very small.

We show in the Fig. 4 the slope of the TF-relations. As we can see the slope steadily increases with increasing distance from the center.

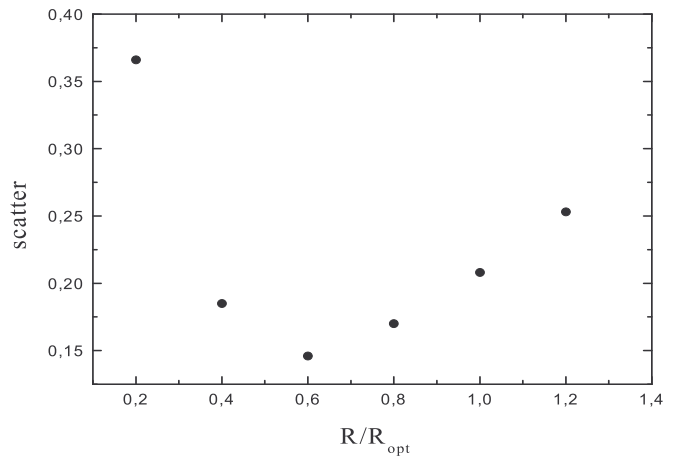

Figure 3. The scatter of the TF-relations as a function of radius.

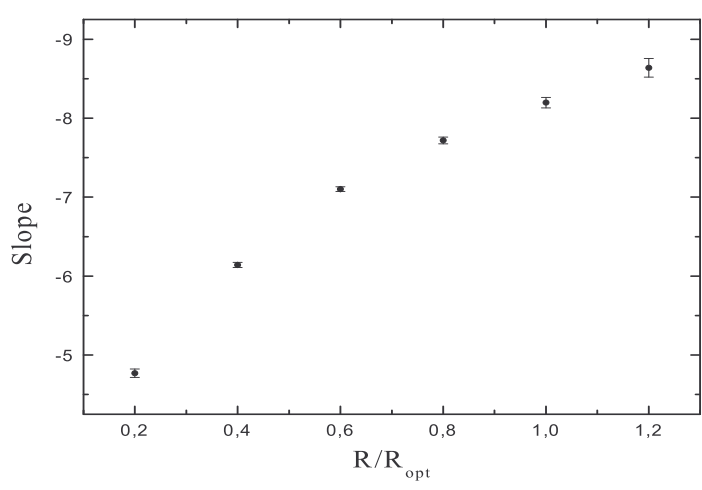

Figure 4. The slope of the TF-relations as a function of radius.

In the Fig. 5 we plot the standard TF-relation and in the Table 2 its parameters. These values are similar to the one at $\mathrm{R}_{\mathrm{opt}}$.

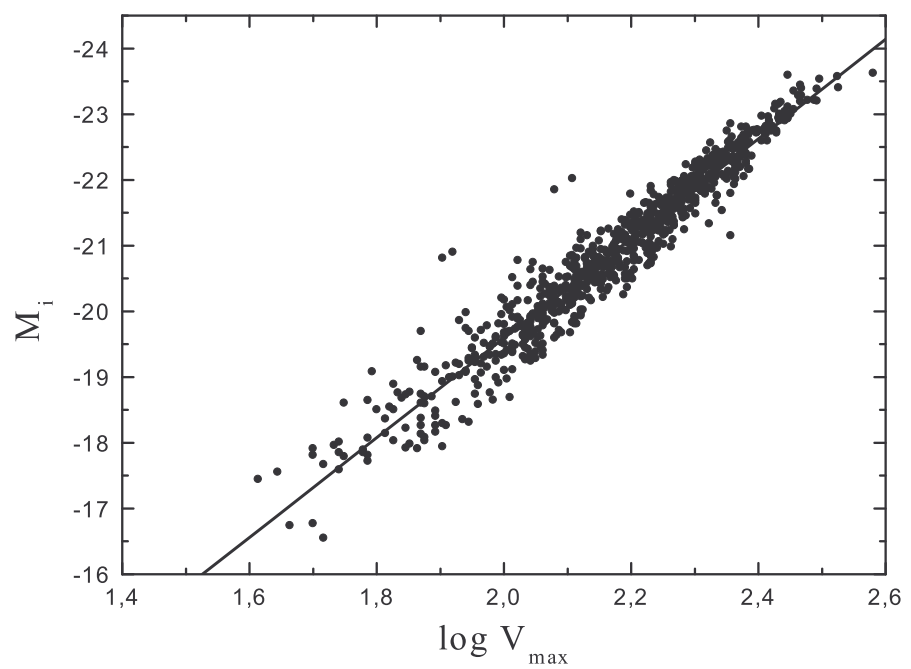

Figure 4. The TF-relations at max velocity. 


\begin{tabular}{|l|l|l|l|l|l|}
\hline $\begin{array}{l}\text { zero } \\
\text { point }\end{array}$ & error & slope & error & SD & N \\
\hline-4.435 & 0.151 & -7.579 & 0.069 & 0.328 & 843 \\
\hline
\end{tabular}

Table 2. The TF-relations at maximum velocity column 1 - intercept value (a)

column 2 - standard error of a

column 3 - slope value (b)

column 4 - standard error of $b$

column 5 - scatter

column 6 - number of points

The first analysis of this data shows that the dark matter fraction depends on the radius and on the galaxy luminosity. Because of this the TF-relations are not parallel to each other (see Fig.2).

We plan to study quantitatively the behavior of scatter and slope in different models of mass distribution.

\section{References}

[1] D.S. Mathewson, V.L. Ford, Parameters of 2447 southern spiral galaxies for use in the TullyFisher relation, 1996, ApJS, 107, 97M

[2] M. Persic, P. Salucci, Rotation curves of 967 spiral galaxies, 1995, ApJS, 99, 105P. 